\title{
Irisin: As a Therapeutic Target for Metabolic Disorders
}

\author{
Ikram Ullah Khan a, b
}

\begin{abstract}
During physical activity, muscle expresses a panel of proteins named as myokines which exert beneficial effects on the distant organs of the body. Irisin is a relatively newly discovered myokine mainly secreted by the skeletal muscles. This myokine is a cleavage fragment of a transmembrane protein known as fibronectin domain-containing protein 5 (FNDC5). Irisin is known to have multi-spectrum functions including browning of adipose tissue (BAT), enhancing insulin sensitivity, cognition, osteogenesis and metabolism. Due to these functions, irisin has a wide range of therapeutic effects on obesity, diabetes mellitus, hypertension, cardiovascular diseases, chronic kidney diseases, cancer and dementia. In current, our focus is emphasized upon the therapeutic aspects of irisin and its possible role in the diagnoses of various metabolic disorders.
\end{abstract}

Keywords: Irisin; FNDC5; Myokine; Obesity; Diabetes; PCOS

\section{Introduction}

Skeletal muscles are one of the important tissues in the body. It can contract and secretes proteins which communicate with other organs via humoral factors. Pedersen and Febbraio proposed that skeletal muscles act as an endocrine organ which produces, expresses and secretes multi-spectrum peptides which exerts an endocrine effect on distant organs and tissues [1]. Such factors have been named as myokine and suggested to play an important role in exercise-mediated metabolic changes. In lean men and women, approximately $40 \%$ of body weight is composed of skeletal muscle mass [2]. After identification of skeletal muscle as an endocrine organ, Pederson and his colleagues analyzed various secreted peptide and identified their natures [2]. Among all these peptides is the brain-derived neurotrophic factor, irisin; interleukins (ILs) including IL-6,

Manuscript submitted September 20, 2018, accepted September 26, 2018

a'Laboratory of Reproductive Neuroendocrinology, Department of Animal Sciences, Faculty of Biological Sciences, Quaid-i-Azam University, Islamabad, Pakistan

${ }^{\mathrm{b}}$ Corresponding Author: Ikram Ullah Khan, Laboratory of Reproductive Neuroendocrinology, Department of Animal Sciences, Faculty of Biological Sciences, Quaid-i-Azam University, Islamabad, Pakistan.

Email: ikram@bs.qau.edu.pk

doi: https://doi.org/10.14740/jem528w
$-15,-8$ and -17 play an important role in homeostasis and insulin signaling [3].

Irisin is a novel peptide of 112 amino acids that boosts metabolism by inducing browning in white adipose tissues (WATs). The primary source of irisin is a skeletal muscle, but some recent studies also show expression of irisin and fibronectin domain-containing protein 5 (FNDC5) mRNA in various human tissues. Besides skeletal muscles, it is also secreted by heart, pancreas, stomach, testis, epididymis, Leydig cells, Kupffer cells, tongue, sinusoidal epithelial cells, rectum, optic nerve, brain and in low levels in kidney, liver and lungs $[4,5]$. Irisin peptide and FNDC5 mRNA have also been observed in the cardiomyocytes, adipose tissue, cerebrospinal fluid and cerebellum in rodents and humans [6, 7]. Another comprehensive study reported irisin in paraventricular neurons of hypothalamus nearby neuropeptide $\mathrm{Y}$ neurons in gestating females, revealing its role in reproduction and puberty [8]. Furthermore, irisin is not only present in serum, but also in saliva and breast milk of lactating women [9]. Other than browning of WAT, various studies have found therapeutic role of irisin, including bone metabolism [10], endothelial cell proliferation [11], diabetes [12-14], obesity [15, 16], polycystic ovarian syndrome [17], and as a metabolic marker in memory cognition, thyroid dysfunction [18], blood pressure [19] and renal dysfunction [20].

Exercise increases the level of peroxisome proliferatoractivated receptor (PPAR) gamma coactivator-1 $\alpha$ (PGC-1 $\alpha$ ). It stimulates the synthesis of FNDC5. The FNDC5 further undergoes cleavage process and secretes irisin hormone into circulation [5]. WATs have many unilocular lipid droplets, a small number of mitochondria in their cells, and store energy in the form of fats [21]. In contrast, brown adipose tissues (BATs) have more mitochondria with uncoupling protein 1 (UCP1) and small multilocular lipid droplets which easily dissipate energy by substrate oxidation [21]. The irisin produced by muscles, converts white adipocytes into beige adipocytes through activating extracellular signal-regulated kinases (ERK) and p38 mitogen-activated protein kinase (MAPK) signaling pathways [5,22]. Due to the phenomenon of browning, irisin can be used as a new therapeutic biomarker of metabolic disorders like obesity, diabetes and glucose intolerance [23-25].

\section{Discovery of irisin}

Muscle-derived factor called irisin was discovered in 2012. The name "irisin" was given after the Greek messenger goddess "Iris" [26]. Irisin is formed by a cleavage of FNDC5. The 
Spiegelman and his colleagues have recently discovered the exercise-induced expression of FNDC5, a type I membrane protein, in the skeletal muscle in a PGC-1 $\alpha$-dependent manner. FNDC5 is released into circulation after proteolytic cleavage as a polypeptide of 115 amino acids termed as irisin, which subsequently converts subcutaneous "beige" fat into "brown" fat [26].

\section{Structure of irisin}

The FNDC5 is a transmembrane glycoprotein. It was first discovered in 2002 [27, 28], and is also called FRCP2 and Pep. Its gene is present on chromosome no. 1 and represented as 1p35.1 (ensemble: ENSG00000160097), encoding a precursor molecule containing 212 amino acids. The precursor molecule contains a signal peptide, fibronectin domains and a hydrophobic domain [27].

The membrane FNDC5 has a $32-\mathrm{kDa}$ molecular weight, which is larger compared to the cellular FNDC5 [28, 29]. In response to the activation of PGC- $1 \alpha$, the FNDC5 proteolytic cleaved into irisin by a protease, is yet to be discovered [26]. After cleavage, the irisin is formed which is an N-terminal portion of FNDC5. It consists of 112 amino acids and has $12-\mathrm{kDa}$ molecular weight. The FNDC5 receptor is essential for the differentiation of myoblasts and neurons, and is highly expressed in pericardium, heart, brain and skeletal muscles [30]. However, the structure of irisin is not yet fully clear. The structure of irisin has $15-20 \%$ homology to FNIII domains and has similar folds. In Escherichia coli, irisin is expressed in mature form and composed of 30 - 140 residues. Irisin possesses structural homology with the third domain of tenascin and the tenth module of fibronectin $[16,21,31,32]$.

\section{Crystalline structure of irisin}

The FNDC5 diagrammatic structure is the irisin subunit structure, indicating the FNIII domain [33]. It suggests that the regions of flexibility existed on the same side and that may be considered as a possible candidate for interact tome interaction region.

\section{Immunocytochemical expression}

Immunocytochemistry suggests the expression of irisin in various tissues of the body. At first, irisin expression was noted in muscle tissues [26]. Apart from the skeletal muscles, it has been reported in various other tissues, including central and peripheral tissues. Centrally, irisin has been localized in the Purkinje cells of the cerebellum [6], brain and spinal cord [4]. In peripheral tissues, it is reported in liver, kidney (Aydin et al, 2014a), salivary glands (Aydin et al, 2013a), cardiac muscles, skin and testis [5]. Furthermore, the mRNA of FNDC5 has been identified in muscle, rectum, pericardium, uvula, thyroid, ovary, pituitary, seminal vesicles, oviduct, testis, urinary bladder and vagina [4].

\section{Irisin receptor}

The receptor of irisin has not yet been identified, but researchers are trying to identify the nature of its receptor [26]. Bostrom et al [26] suggested the existence of a cell surface receptor, whereas Schumacher et al [33] observed a dimer structure of irisin, which may be essential for ligand-receptor binding. Furthermore, Schumacher and his colleagues [33] suggested the loop 106 - 108 of irisin as a possible region for interaction with the unidentified receptor.

\section{Circulating levels of irisin in human}

Irisin has been reported from the plasma of rat [23], mice [26] and human $[4,13,16,26]$ using enzyme-linked immunosorbent assay (ELISA) [32]. Several studies reported circulating level of irisin in human. However, under the condition of obesity, the results were controversial. Some of the studies found a negative association between irisin level and obesity [16]. Currently, no such association has been reported between these two parameters in humans [34], except for the decreased in irisin level after surgery and dietary-induced weight loss [14, 35].

\section{Functions}

Irisin is responsible for multi-spectrum functions, such as metabolic activities and physiological actions carried out in various body organs [36]. The circulating irisin positively associate with the circumference of bicep muscles, fat-free mass, glucose, ghrelin and insulin-like growth factor I (IGF-I), but negatively correlate with age, insulin and adiponectin $[4,13$, 16].

\section{Browning}

According to Bostrom and his colleagues [26], irisin plays an important role in reducing obesity by converting white adipocytes into brown adipocytes. In the same experiment, they observed enhanced oxygen consumption in differentiating adipocytes after injecting FNDC5. The irisin down-regulated the specific genes of WAT. Moreover, a moderate increase in the plasma irisin causes an increase energy expenditure and insulin resistance in obese mice $[26,36]$. Finally, it was demonstrated that exercise-induced irisin is involved in the browning of white fat and concluded that the rise in irisin is mediated by augmented concentrations of PGC-1 $\alpha$ in muscle, while PPAR- $\alpha$ acts as the downstream target of this hormone [37].

Roca-Rivada and his coworkers [7] suggest that apart from skeletal muscles, irisin is also synthesized and secreted by the adipose tissues, called "adipokine" that acts as an endocrine and autocrine factor. Moreover, they also showed that irisin has a different pattern of secretion depending upon the location of adipose tissue. Thus, visceral adipose tissue secretes lower irisin than subcutaneous, reflecting one more time that visceral 
fat is more implicated in metabolic complications, while subcutaneous fat has a possible beneficial role. They also showed that acute exercise training induces FNDC5 secretion in obese, but this secretion was significantly reduced in fasting animals. The increased secretion of irisin in obese animals suggested a type of hormonal resistance. Furthermore, Bostrom and his coworkers [26] observed that irisin acts as a down-regulator of white adipocytes genes, so we can suggest that irisin can be used as a therapeutic target for obesity on the molecular level.

\section{Cognition}

Besides the role of irisin in adipose tissue and muscle, FNDC5 and irisin expression is also speculated in some parts of the brain. It suggests that FNDC5 is required for adequate neural differentiation of mouse embryonic stem cells [6]. Mouse embryonic stem cell knockdowns for FNDC5 have shown reduced neuronal differentiation and disturbed post-neuronal progenitor formation. Additionally, a novel neural pathway is hypothesized, where irisin produced in the cerebellum through several transitional pathways in spinal cord and medulla, regulates thermogenesis [38]. However, this striking feature of irisin still needs to be explored.

Finally, Moon and his colleagues [39] observed enhanced hippocampal neuronal cell proliferation and differentiation in a dose-dependent manner by increasing the pharmacological concentrations of irisin. Although the underlying molecular mechanism needs to be confirmed, it is considered to occur through signal transducer and activator of transcription 3 (STAT3) and signal transducers rather than extracellular signal-regulated kinase (ERK) and adenosine monophosphateactivated protein kinase (AMPK) signal transduction pathways $[5,22]$.

Exercise selectively induces hippocampal $P g c-1 \alpha$ and Fndc5 gene expression in mouse brain [40]. It suggests that irisin is one of the possible candidate markers involved in regulating the neurogenesis, and may be used as a therapeutic agent for various neurodegenerative diseases, including Alzheimer's disease, Parkinson's disease, etc.

\section{Function in heart}

The cardiac myoblasts contain more irisin than the skeletal muscles, where it improves cardiac functions. Different studies revealed irisin as a strong modulator of cardio-myoblast performance. It enhances mitochondrial thermogenesis and calcium signaling, where the former is required for mechanical work and the latter is responsible for electrical conductivity and contractions of cardiac muscle. Exercise-induced irisin levels have a beneficial effect on the cardio-myoblasts by increasing oxygen consumption and regulating intracellular calcium concentrations. Cardio-myoblasts membrane contains irisin receptors where through extracellular signal-regulated kinases ERK and MAPK signal transduction, it contributes to a variety of cellular responses like cell growth, proliferation, differentiation and even apoptosis [41].
Administration of irisin to human umbilical vein endothelial cells enhances proliferation. This correlates with the heart tissue development and remodeling, while the mechanism needs to be determined [42]. Further studies are required to understand the mechanistic role of irisin in contractility of cardiac muscle and impulse propagation.

\section{Function in thyroid gland}

Irisin has a potent effect on metabolism and thermogenesis by controlling the levels of thyroid hormones. Thyroid hormones are also involved in energy production and thermoregulation. Thyroxine and metabolically active triiodothyronine by influencing BAT, generate heat and regulate energy balance by activating various metabolic processes $[43,44]$. Although the information is scarce in hypothyroid patients, the FNDC5 gene is said to be expressed in thyroid gland cells, which discloses a connection between irisin levels and thyrometabolism. Stengel and his colleagues [16] suggested correlation between thyroidstimulating hormone (TSH) and irisin hormone in obese and anorectic patients.

On the other hand, Ates and his colleagues [45] documented a positive correlation of irisin level with TSH and negatively with free thyroxin (fT4), suggesting that irisin and fT4 are independent risk factors for hypothyroidism. In the case of hyperthyroidism, due to reduction in hypothalamic secretion, BAT thermogenesis is affected. Both conditions of hyper- and hypothyroidism have a negative impact on muscle physiology [46]. In conclusion, the irisin being as a metabolic marker, its biosynthesis is affected in conditions of hypo- and hyperthyroidism. This area required further studies to decipher the role of irisin in hypo- and hyperthyroidism conditions.

\section{Role in osteoblast differentiation}

There is a strong cross-link between the skeletal muscles and bones. The factors secreted by skeletal muscles act on bone via paracrine manners and affect the bone physiology. Notably, the myokines secreted by the skeletal muscle are involved in the healing process of bone. The hypothesis that muscle supports bone mass is proven by Karasik and Kiel [47] through microgravity, and bed rest studies [48], as well as the results of various studies indicate that osteoporosis and sarcopenia are associated at the same time. Physical activity is involved in the development of muscle, bone mass, and enhances weightbearing skeleton. The exercise increases the bone strength, and this is supported in tennis players for having high bone strength and mass in their playing arm in comparison to the non-playing arm [49]. In contrast, the absence of physical activity deforms the body shape and disuses the muscles, and body becomes a target for various pathological conditions, leading to severe bone loss [50]. It suggests that under the condition of physical activity, the myotubes and myoblasts secrete various factors, which enhances the differentiation of osteoblast. It can also be suggested that the increase in differentiation might be due to the presence of irisin receptors on the osteoblast. There- 
fore, we can say that skeletal muscle through physical activity strengthens the bone, which is mediated via irisin. Based on these findings, we can hypothesize that irisin may be a direct regulator of bone metabolism and enhance the differentiation of bone marrow stromal cells to mature osteoblasts. We can relate this hypothesis with the experiment in which one group of mice was subjected to exercise for 3 weeks of free wheel running and other group remained in a non-exercised state. The conditioned media (CM) were collected from myoblast of both groups and it was observed that differentiation of osteoblast was greater in exercising group. Also, there was an enhanced expression of alkaline phosphatase and collagen I through irisin-dependent mechanism [10]. Therefore, we suggest that irisin might be a direct regulator of osteoblast differentiation.

\section{Role in pregnancy and infants}

The presence of irisin is also speculated in the cord blood, where it may promote adequate proliferation and differentiation of different stem cells [51]. Newborn infants have a higher content of BAT required for thermogenesis and its percentage declines after infancy. Appropriate gestational age (AGA) infants have higher total body fat percentage as compared to smaller gestational age (SGA) infants which is a consequence of lower irisin level $[52,53]$. A cross-sectional study conducted by Caglar and his colleagues [54] explained the relation between cord blood irisin level and birth weight. Furthermore, they reported a direct correlation of irisin level with gestational age and fetal weight. A significantly decreased level of irisin is responsible for restricted growth in infants. Similarly, a detectable irisin level is also present in the maternal circulation. Its lower level correlates with the gestational diabetes mellitus, preeclampsia and other complications of pregnancy [8].

\section{Regulation of blood pressure}

The activation of the paraventricular (PVN) neurons increased the blood pressure after administration of recombinant irisin through intra-cerebro-ventricular (ICV) injection. The central administration is associated with an increase in cardiac contractility, but the peripheral administration is associated with the inhibition of cardiac contractibility [19]. The mechanism is not completely clear yet; however, it is considered that it might be due to the coordination between central nervous system and cardiovascular system. From these studies, we can suggest that central irisin may have functioned to increase the cardiac output and blood pressure by regulating the hypothalamic PVN neurons.

\section{Irisin and Metabolic Disorders}

\section{Irisin and obesity}

According to the World Health Organization (WHO), obesity is one of the endemic diseases [55] and considered as a common nutritional disease in the various countries. Obesity is associated with other diseases, including diabetes [56], cardiovascular disease and a certain form of respiratory problems. Obesity develops when more energy is stored in the body as compared to the energy expenditure through metabolism, physical activities, body maintenance and thermogenesis. A person becomes obese through the accumulation of WAT that is specialized to store energy in the body in the form of lipids, known as triglycerides. The BAT is involved in the metabolism of glucose and fatty acids, yielding heat energy, thus contributing towards thermogenic activity $[26,35]$. Such thermogenic activity of BAT is possible due to a specific uncoupling protein (UCP1) in its mitochondria. The UCP1 disengages the mitochondrial proton gradient from ATP synthesis and produces heat. In addition, the exercise-induced myokine, such as irisin, is involved in the thermogenesis of WAT into BAT and mediates weight loss [36]. Thus, due to the interacted property of weight loss, irisin can be used as a therapeutic target for obesity.

\section{Irisin and diabetes}

In addition to the role of irisin in the browning of adipocytes, many studies have investigated the therapeutic action of iri$\sin$ in diabetes [14]. The accumulation of adipose tissues in the muscle hides the insulin receptors [57], which increases insulin resistance. Irisin increases the browning [35], causing a reduction of white fat. It can be possible to use irisin as a therapeutic target for diabetes to reduce the insulin resistance.

\section{Gestational diabetes mellitus (GDM)}

GDM is a physiological condition of glucose intolerance in pregnancy [58]. It has serious adverse effects, such as enhancing long-term risk of obesity, diabetes and increased risk of maternal and fetal morbidity [59]. Insulin resistance is the major cause of GDM, but the controlling mechanism is not yet fully understood. According to our knowledge, this is the first study in which the maternal serum irisin levels were significantly lower in pregnant women with GDM compared to control group [13]. Similarly, Kuzmicki and his colleagues [60] found increased level of serum irisin in women during pregnancy, but lower in women with GDM. The low level of irisin under GDM notably seeks our attention that irisin being as a metabolic marker may show an adaptive response to compensate energy expenditure in GDM.

\section{Irisin and polycystic ovarian syndrome (PCOS)}

Low irisin levels have been detected in the amenorrhoeic athletes compared to the eumenorrheic athletes and non-athletes [16]. Other studies reported an association of irisin in the pathological condition and suggested a positive association of irisin in PCOS. The PCOS is a reproductive endocrinopathy, characterized by menstrual dysfunction and insulin resistance (IR). The irisin level is raised in patients with PCOS [17, 61, 62]; 
however, a recent study indicated low irisin level in PCOS [13].

These findings notably seek our attention towards an adaptive response of irisin to compensate energy expenditure in amenorrhoeic athletes and PCOS patients, as reproduction itself is an energy-demanding process. Hence, these studies depict that irisin might be a peripheral signal to integrate with reproductive pathways in physiological as well as pathological conditions.

\section{Conclusions}

The currently discovered muscle secreted factor, irisin, has an essential role in the treatment of various metabolic disorders. The irisin has multiple effects on the browning of white adipocytes. Previous studies demonstrated the role of recombinant FNDC5 and irisin in inducing browning of WAT. However, there is a lack of understanding about the data in human that recombinant FNDC5 and irisin can induce browning in human adipose tissues. Recently, studies conducted in human have suggested that a type of exercise, whether acute or chronic, has no such effect on the circulating levels of FNDC5 and irisin. This lack of correlation between a type of physical activity and irisin level depends upon the type of antibodies, condition of the experiment and the type of model organisms used in these studies. Being as a metabolic marker, irisin can be used as a therapeutic target for various metabolic diseases.

\section{References}

1. Pedersen BK, Steensberg A, Fischer C, Keller C, Keller P, Plomgaard P, Febbraio M, et al. Searching for the exercise factor: is IL-6 a candidate? J Muscle Res Cell Motil. 2003;24(2-3):113-119.

2. Pedersen BK, Febbraio MA. Muscles, exercise and obesity: skeletal muscle as a secretory organ. Nat Rev Endocrinol. 2012;8(8):457-465.

3. Pedersen BK. Muscular interleukin-6 and its role as an energy sensor. Med Sci Sports Exerc. 2012;44(3):392396.

4. Huh JY, Panagiotou G, Mougios V, Brinkoetter M, Vamvini MT, Schneider BE, Mantzoros CS. FNDC5 and irisin in humans: I. Predictors of circulating concentrations in serum and plasma and II. mRNA expression and circulating concentrations in response to weight loss and exercise. Metabolism. 2012;61(12):1725-1738.

5. Aydin S, Kuloglu T, Aydin S, Kalayci M, Yilmaz M, Cakmak T, Albayrak S, et al. A comprehensive immunohistochemical examination of the distribution of the fat-burning protein irisin in biological tissues. Peptides. 2014;61:130-136.

6. Dun SL, Lyu RM, Chen YH, Chang JK, Luo JJ, Dun NJ. Irisin-immunoreactivity in neural and non-neural cells of the rodent. Neuroscience. 2013;240:155-162.

7. Roca-Rivada A, Castelao C, Senin LL, Landrove MO, Baltar J, Belen Crujeiras A, Seoane LM, et al. FNDC5/ irisin is not only a myokine but also an adipokine. PLoS One. 2013;8(4):e60563.
8. Piya MK, Harte AL, Sivakumar K, Tripathi G, Voyias PD, James S, Sabico S, et al. The identification of irisin in human cerebrospinal fluid: influence of adiposity, metabolic markers, and gestational diabetes. Am J Physiol Endocrinol Metab. 2014;306(5):E512-518.

9. Aydin S, Kuloglu T, Aydin S. Copeptin, adropin and irisin concentrations in breast milk and plasma of healthy women and those with gestational diabetes mellitus. Peptides. 2013;47:66-70.

10. Colaianni G, Cuscito C, Mongelli T, Oranger A, Mori G, Brunetti G, Colucci S, et al. Irisin enhances osteoblast differentiation in vitro. Int J Endocrinol. 2014;2014:902186.

11. Song H, Wu F, Zhang Y, Zhang Y, Wang F, Jiang M, Wang $Z$, et al. Irisin promotes human umbilical vein endothelial cell proliferation through the ERK signaling pathway and partly suppresses high glucose-induced apoptosis. PLoS One. 2014;9(10):e110273.

12. Choi YK, Kim MK, Bae KH, Seo HA, Jeong JY, Lee WK, Kim JG, et al. Serum irisin levels in new-onset type 2 diabetes. Diabetes Res Clin Pract. 2013;100(1):96-101.

13. Liu JJ, Wong MD, Toy WC, Tan CS, Liu S, Ng XW, Tavintharan $\mathrm{S}$, et al. Lower circulating irisin is associated with type 2 diabetes mellitus. J Diabetes Complications. 2013;27(4):365-369.

14. Moreno-Navarrete JM, Ortega F, Serrano M, Guerra E, Pardo G, Tinahones F, Ricart W, et al. Irisin is expressed and produced by human muscle and adipose tissue in association with obesity and insulin resistance. J Clin Endocrinol Metab. 2013;98(4):E769-778.

15. Timmons JA, Baar K, Davidsen PK, Atherton PJ. Is irisin a human exercise gene? Nature. 2012;488(7413):E9-10; discussion E10-11.

16. Stengel A, Hofmann T, Goebel-Stengel M, Elbelt U, Kobelt P, Klapp BF. Circulating levels of irisin in patients with anorexia nervosa and different stages of obesitycorrelation with body mass index. Peptides. 2013;39:125130.

17. Chang CL, Huang SY, Soong YK, Cheng PJ, Wang CJ, Liang IT. Circulating irisin and glucose-dependent insulinotropic peptide are associated with the development of polycystic ovary syndrome. J Clin Endocrinol Metab. 2014;99(12):E2539-2548.

18. Samy DM, Ismail CA, Nassra RA. Circulating irisin concentrations in rat models of thyroid dysfunction - effect of exercise. Metabolism. 2015;64(7):804-813.

19. Zhang W, Chang L, Zhang C, Zhang R, Li Z, Chai B, Li $\mathrm{J}$, et al. Central and peripheral irisin differentially regulate blood pressure. Cardiovasc Drugs Ther. 2015;29(2):121127.

20. Ebert T, Focke D, Petroff D, Wurst U, Richter J, Bachmann A, Lossner U, et al. Serum levels of the myokine irisin in relation to metabolic and renal function. Eur J Endocrinol. 2014;170(4):501-506.

21. Cannon B, Nedergaard J. Brown adipose tissue: function and physiological significance. Physiol Rev. 2004;84(1):277-359.

22. Jones SA, Scheller J, Rose-John S. Therapeutic strategies for the clinical blockade of IL-6/gp130 signaling. J Clin Invest. 2011;121(9):3375-3383. 
23. Sharma N, Castorena CM, Cartee GD. Greater insulin sensitivity in calorie restricted rats occurs with unaltered circulating levels of several important myokines and cytokines. Nutr Metab (Lond). 2012;9(1):90.

24. Hojlund K, Bostrom P. Irisin in obesity and type 2 diabetes. J Diabetes Complications. 2013;27(4):303-304.

25. Vamvini MT, Aronis KN, Panagiotou G, Huh JY, Chamberland JP, Brinkoetter MT, Petrou M, et al. Irisin mRNA and circulating levels in relation to other myokines in healthy and morbidly obese humans. Eur J Endocrinol. 2013;169(6):829-834.

26. Bostrom P, Wu J, Jedrychowski MP, Korde A, Ye L, Lo JC, Rasbach KA, et al. A PGC1-alpha-dependent myokine that drives brown-fat-like development of white fat and thermogenesis. Nature. 2012;481(7382):463-468.

27. Ferrer-MartinezA, Ruiz-Lozano P, Chien KR. Mouse PeP: a novel peroxisomal protein linked to myoblast differentiation and development. Dev Dyn. 2002;224(2):154-167.

28. Teufel A, Malik N, Mukhopadhyay M, Westphal H. Frcp1 and Frcp2, two novel fibronectin type III repeat containing genes. Gene. 2002;297(1-2):79-83.

29. Leahy DJ, Hendrickson WA, Aukhil I, Erickson HP. Structure of a fibronectin type III domain from tenascin phased by MAD analysis of the selenomethionyl protein. Science. 1992;258(5084):987-991.

30. Aydin S, Aydin S, Kuloglu T, Yilmaz M, Kalayci M, Sahin I, Cicek D. Alterations of irisin concentrations in saliva and serum of obese and normal-weight subjects, before and after 45 min of a Turkish bath or running. Peptides. 2013;50:13-18.

31. Leahy DJ, Aukhil I, Erickson HP. 2.0 A crystal structure of a four-domain segment of human fibronectin encompassing the RGD loop and synergy region. Cell. 1996;84(1):155-164.

32. Handschin C, Spiegelman BM. The role of exercise and PGC1alpha in inflammation and chronic disease. Nature. 2008;454(7203):463-469.

33. Schumacher MA, Chinnam N, Ohashi T, Shah RS, Erickson HP. The structure of irisin reveals a novel intersubunit beta-sheet fibronectin type III (FNIII) dimer: implications for receptor activation. J Biol Chem. 2013;288(47):33738-33744.

34. Crujeiras AB, Pardo M, Arturo RR, Navas-Carretero S, Zulet MA, Martinez JA, Casanueva FF. Longitudinal variation of circulating irisin after an energy restrictioninduced weight loss and following weight regain in obese men and women. Am J Hum Biol. 2014;26(2):198-207.

35. Sanchis-Gomar F, Alis R, Pareja-Galeano H, Sola E, Victor VM, Rocha M, Hernandez-Mijares A, et al. Circulating irisin levels are not correlated with BMI, age, and other biological parameters in obese and diabetic patients. Endocrine. 2014;46(3):674-677.

36. Xu X, Ying Z, Cai M, Xu Z, Li Y, Jiang SY, Tzan K, et al. Exercise ameliorates high-fat diet-induced metabolic and vascular dysfunction, and increases adipocyte progenitor cell population in brown adipose tissue. Am J Physiol Regul Integr Comp Physiol. 2011;300(5):R1115-1125.

37. Erickson HP. Irisin and FNDC5 in retrospect: An exercise hormone or a transmembrane receptor? Adipocyte.
2013;2(4):289-293.

38. Hashemi MS, Ghaedi K, Salamian A, Karbalaie K, Emadi-Baygi M, Tanhaei S, Nasr-Esfahani MH, et al. Fndc5 knockdown significantly decreased neural differentiation rate of mouse embryonic stem cells. Neuroscience. 2013;231:296-304.

39. Moon HS, Dincer F, Mantzoros CS. Pharmacological concentrations of irisin increase cell proliferation without influencing markers of neurite outgrowth and synaptogenesis in mouse H19-7 hippocampal cell lines. Metabolism. 2013;62(8):1131-1136.

40. Wrann CD, White JP, Salogiannnis J, Laznik-Bogoslavski D, Wu J, Ma D, Lin JD, et al. Exercise induces hippocampal BDNF through a PGC-1alpha/FNDC5 pathway. Cell Metab. 2013;18(5):649-659.

41. Emanuele E, Minoretti P, Pareja-Galeano H, SanchisGomar F, Garatachea N, Lucia A. Serum irisin levels, precocious myocardial infarction, and healthy exceptional longevity. Am J Med. 2014;127(9):888-890.

42. Chaanine AH, Hajjar RJ. AKT signalling in the failing heart. Eur J Heart Fail. 2011;13(8):825-829.

43. Raschke S, Eckel J. Adipo-myokines: two sides of the same coin - mediators of inflammation and mediators of exercise. Mediators Inflamm. 2013;2013:320724.

44. Lahesmaa M, Orava J, Schalin-Jantti C, Soinio M, Hannukainen JC, Noponen T, Kirjavainen A, et al. Hyperthyroidism increases brown fat metabolism in humans. J Clin Endocrinol Metab. 2014;99(1):E28-35.

45. Ates I, Altay M, Topcuoglu C, Yilmaz FM. Circulating levels of irisin is elevated in hypothyroidism, a case-control study. Arch Endocrinol Metab. 2016;60(2):95-100.

46. Rooyackers OE, Nair KS. Hormonal regulation of human muscle protein metabolism. Annu Rev Nutr. 1997;17:457485.

47. Karasik D, Kiel DP. Evidence for pleiotropic factors in genetics of the musculoskeletal system. Bone. 2010;46(5):1226-1237.

48. LeBlanc AD, Spector ER, Evans HJ, Sibonga JD. Skeletal responses to space flight and the bed rest analog: a review. J Musculoskelet Neuronal Interact. 2007;7(1):3347.

49. Jones HH, Priest JD, Hayes WC, Tichenor CC, Nagel DA. Humeral hypertrophy in response to exercise. J Bone Joint Surg Am. 1977;59(2):204-208.

50. Rodriguez JI, Palacios J, Garcia-Alix A, Pastor I, Paniagua R. Effects of immobilization on fetal bone development. A morphometric study in newborns with congenital neuromuscular diseases with intrauterine onset. Calcif Tissue Int. 1988;43(6):335-339.

51. Ojha S, Robinson L, Yazdani M, Symonds ME, Budge H. Brown adipose tissue genes in pericardial adipose tissue of newborn sheep are downregulated by maternal nutrient restriction in late gestation. Pediatr Res. 2013;74(3):246251.

52. Joung KE, Park KH, Filippaios A, Dincer F, Christou H, Mantzoros CS. Cord blood irisin levels are positively correlated with birth weight in newborn infants. Metabolism. 2015;64(11):1507-1514.

53. van de Lagemaat M, Rotteveel J, Lafeber HN, van Weis- 
senbruch MM. Lean mass and fat mass accretion between term age and 6 months post-term in growth-restricted preterm infants. Eur J Clin Nutr. 2014;68(11):1261-1263.

54. Caglar M, Goksu M, Isenlik BS, Yavuzcan A, Yilmaz M, Ustun Y, Aydin S, et al. Irisin in idiopathic foetal growth restriction. J Endocrinol Invest. 2014;37(7):619-624.

55. James WP. WHO recognition of the global obesity epidemic. Int J Obes (Lond). 2008;32(Suppl 7):S120-126.

56. Park KH, Zaichenko L, Brinkoetter M, Thakkar B, SahinEfe A, Joung KE, Tsoukas MA, et al. Circulating irisin in relation to insulin resistance and the metabolic syndrome. J Clin Endocrinol Metab. 2013;98(12):4899-4907.

57. Kadowaki T, Yamauchi T, Kubota N, Hara K, Ueki K, Tobe K. Adiponectin and adiponectin receptors in insulin resistance, diabetes, and the metabolic syndrome. J Clin Invest. 2006;116(7):1784-1792.

58. Catalano PM, Kirwan JP, Haugel-de Mouzon S, King J. Gestational diabetes and insulin resistance: role in shortand long-term implications for mother and fetus. J Nutr.
2003;133(5 Suppl 2):1674S-1683S.

59. Xiong $X$, Saunders LD, Wang FL, Demianczuk NN. Gestational diabetes mellitus: prevalence, risk factors, maternal and infant outcomes. Int J Gynaecol Obstet. 2001;75(3):221-228.

60. Kuzmicki M, Telejko B, Lipinska D, Pliszka J, Szamatowicz M, Wilk J, Zbucka-Kretowska M, et al. Serum irisin concentration in women with gestational diabetes. Gynecol Endocrinol. 2014;30(9):636-639.

61. Pukajlo K, Laczmanski L, Kolackov K, KuliczkowskaPlaksej J, Bolanowski M, Milewicz A, Daroszewski J. Irisin plasma concentration in PCOS and healthy subjects is related to body fat content and android fat distribution. Gynecol Endocrinol. 2015;31(11):907-911.

62. Li M, Yang M, Zhou X, Fang X, Hu W, Zhu W, Wang C, et al. Elevated circulating levels of irisin and the effect of metformin treatment in women with polycystic ovary syndrome. J Clin Endocrinol Metab. 2015;100(4):14851493. 